angle between the line joining them and their rotation axes is about $50^{\circ} \mathrm{II}^{\prime}$. So far as the translational forces are concerned the surprising result is obtained that electrostatic forces alone are sufficient to produce this stable equilibrium, that is merely by the use of the inverse square law between infinitesimal elements of charge. To obtain this result, however, requires that the ratio of the major to the minor axes of the electrons shall be about three to one. This is also the shape assigned to the electrons on other grounds in the previous abstracts cited, and, hence, the two independent results support each other.

This result is, therefore, independent of the special form of electromagnetic theory employed, whether the Saha theory or that of Lorentz. It requires between 10,000 and 100,000 such atoms strung together to bridge the distance ${ }^{10^{-8}} \mathrm{~cm}$. This means that there is plenty of room for the smallest dimension of the molecule to be of the order of $10^{-8}, \mathrm{IO}^{-9}, \mathrm{IO}^{-10}, \mathrm{IO}^{-11}$ or even $1 \mathrm{O}^{-12} \mathrm{~cm}$., and it has been difficult to understand why $10^{-8} \mathrm{~cm}$. is the order selected by practically all forms of atoms as the smallest dimension of its molecules approximately speaking. The above example, which is typical of all, attributes this particular value $\mathrm{IO}^{-8} \mathrm{~cm}$. to the shape of the electron itself. Granting that this is the truth of the matter, the universal presence of electrons as parts of all atoms makes it reasonable that $\mathrm{IO}^{-8} \mathrm{~cm}$. should be a universal order of distance.

The secret of obtaining any result by the use of electrostatic forces so far as translational forces are concerned is connected with the kind of atom used, in which the positive and the negative electrons are already in equilibrium because of their very close proximity to each other.

Cleveland, Ohio,

November 7, I921.

\title{
The Classification and the Prediction of Isotopes.
}

By William D. Harkins.

An earlier paper in this Review (15, 73-94 (1920)) pointed out the fact that nearly all known atoms contain an even number of negative nuclear electrons. Atom nuclei may be divided into four classes. Ninety per cent. of all known atoms belong to Class I., in which the number $(P)$ of positive electrons and the number $(N)$ of negative nuclear electrons are both even. In Class II. $N$ is even and $P$ is odd and in Class III. both are odd. Almost no atoms of Class IV. exist either on earth or in the meteorites. In this class $N$ is odd and $P$ is even. In I9r 5 Harkins and Wilson developed the following equation for the atomic weights of the atomic species:

$$
P=2(M+f),
$$

in which $P$ designates the atomic weight, $M$ is the atomic number, and $f$ varies in steps of $\frac{1}{2}$ from - I to 27 , but for complex nuclei only from o to 27 . The value of $f$ gives the number of cementing electrons for all atoms whose 
atomic weight is divisible by 4 . The values of $2 f$ are more suited for use as the basis of a system of classification, since they include only every whole number from o to 57 , if only complex nuclei are considered. The number $2 f$ may be designated as the isotopic number $(n)$. Atoms may be classified also according to the disintegration or aggregation series to which they belong. The paper will consider a nomenclature of the radioactive and other isotopes based upon these systems of classification.

The principles previously developed indicate that in general the number of isotopes of elements of even number is considerably greater than for elements of odd atomic number, particularly for elements whose atomic number is greater than thirty. The most abundant isotope and most of the other isotopes of an element of even atomic number, have atomic weights which are even numbers, while if the atomic number is odd the atomic weights of the isotopes are almost always odd. If the chemical atomic weight is known with a high degree of precision, most of the isotopes may be predicted by the use of these and other relations.

University of Chicago.

\section{The Nature of Charcoal "Sorption."}

\section{By H. HoRton SHeldon.}

IT has been pointed out by Dr. J. W. McBain that charcoal "sorption" of gases can be divided into two parts, adsorption on the surface and absorption into the interior. The adsorption he assumes to take place according to the theory suggested by Dr. Langmuir but the absorption he considers to be solid solution, and extends this to include the case of "sorption" of iodine from solutions of benzene, etc. ${ }^{2}$

Plotting the pressure-time curve for gases, or the concentration time-curve for solutions, there are evidently two distinct parts to the curve, separated by a bend. That this actually exists can be shown by plotting the logarithms of the numbers used, in which case the bend is such as to be unmistakable.

This undoubtedly means a dual action, the second beginning however only after the first is well on its way toward completion. Since solution should begin as soon as adsorption does, the bend is not accounted for and accordingly it is suggested that the second part is due to diffusion into the more inaccessible channels, which cannot be expected to take place to any extent until the easily accessible or directly exposed portions are saturated.

UnIVERSity OF Michigan.

\section{The Scattering of Electrons by Nickel.}

By C. Davisson and C. H. Kunsman.

THE emission from a nickel target under bombardment by electrons has been found to contain a small fraction of electrons with speeds comparable

1 Phil. Mag., 6, I8, 9I6, I909.

2 Trans. Faraday Soc., XIV., 3, 202, I9I9. 